\title{
Pressure gradient measurement using phase contrast (PC)-MRI in stenotic phantom models: Towards noninvasive quantification of fractional flow reserve in the coronary arteries
}

\author{
Zixin Deng ${ }^{1,2^{*}}$, Sang Eun Lee ${ }^{3}$, Zhaoyang Fan ${ }^{1}$, Christopher T Nguyen ${ }^{1}$, Qi Yang ${ }^{1}$, Xiaoming Bi ${ }^{4}$, \\ Byoung Wook Choi ${ }^{5}$, Daniel S Berman ${ }^{1}$, Hyuk-Jae Chang ${ }^{3}$, Debiao Li, ${ }^{1,2}$
}

From 19th Annual SCMR Scientific Sessions

Los Angeles, CA, USA. 27-30 January 2016

\section{Background}

Fractional flow reserve (FFR) is an invasive diagnostic tool to evaluate the functional significance of an intermediate coronary stenosis by quantifying the pressure gradient $(\Delta \mathrm{P})$ across that stenosis [1]. Noninvasive $\Delta \mathrm{P}$ measurement $\left(\Delta \mathrm{P}_{\mathrm{MR}}\right)$ using phase-contrast (PC)-MRI in conjunction with Navier-Stokes (NS) equations has been attempted in large to medium size vessels [2-4], and recently been applied to smaller coronary arteries [5]. However, the measurement accuracy awaits systematic validation given that the small, tortuous, and moving caliber in the coronary artery, particularly when a stenosis exists, may elicit errors in flow velocity quantification by PC-MRI. This study aimed to investigate the feasibility of deriving $\Delta \mathrm{P}_{\mathrm{MR}}$ in small caliber stenotic phantom models at various diameters and its correlation with measured $\Delta \mathrm{P}$ values via a pressure transducer $\left(\Delta \mathrm{P}_{\mathrm{PT}}\right)$.

\section{Methods}

11 small caliber phantom models ranging from $0 \%-85 \%$ area stenosis, with a reference diameter of $4.8 \mathrm{~mm}$, were individually connected to a flow pump (gadoliniumdoped water, constant volume velocity $=250 \mathrm{~mL} / \mathrm{min}$ ) while 2D cross-sectional PC-MRI images were acquired. Contiguous slices (10-20) were consecutively collected across each narrowing (fig. $1 \mathrm{a} / \mathrm{b}$ ). Imaging parameters were: $\mathrm{FA}=15^{\circ}$; $\mathrm{TE} / \mathrm{TR}=\sim 4.0 / 70 \mathrm{~ms}$; in-plane spatial resolution $=\sim 0.55 \times 0.55 \mathrm{~mm}^{2}$; slice thickness $=3.2 \mathrm{~mm}$; $\mathrm{V}_{\text {enc }}=\mathrm{z}(40-260 \mathrm{~cm} / \mathrm{s})$ and $\mathrm{x}, \mathrm{y}(40-80 \mathrm{~cm} / \mathrm{s})$, depending

${ }^{1}$ Cedars Sinai Medical Center, Los Angeles, CA, USA

Full list of author information is available at the end of the article on the degree of narrowing. Eddy-current correction was done offline followed by NS calculations [6]. Repeat scans were performed in $7 / 11$ phantom models and reproducibility was assessed by calculating the intra-class correlation coefficient (ICC) and Bland-Altman plots. Immediately following the PC-MRI scans, pressure was measured using an arterial catheter connected to a pressure transducer at $\sim 1.5 \mathrm{~cm}$ before and $\sim 3 \mathrm{~cm}$ after the maximum narrowing of the phantom models.

\section{Results}

Bland-Altman plots of peak velocities and $\Delta \mathrm{P}_{\mathrm{MR}}$ are shown in fig. 2a. For velocity measurements, excellent correlation was seen in the through-plane peak velocities $(\mathrm{Vz}, \mathrm{ICC}=0.90)$ and lower in Vx $(\mathrm{ICC}=0.57)$ and $\mathrm{Vy}$ $(\mathrm{ICC}=0.58)$. For $\Delta \mathrm{P}_{\mathrm{MR}} \mathrm{s}$, overall $\mathrm{ICC}=0.87$; When observed individually, higher correlation was seen at smaller stenosis degrees and weaker as stenosis increased (fig. 1b). This could be due to the increased velocity in larger stenosis, causing minor turbulence distal of the narrowing, thus, inconsistent velocity and $\Delta \mathrm{P}_{\mathrm{MR}}$ between the two scans. Furthermore, $\Delta \mathrm{P}_{\mathrm{MR}}$ and $\Delta \mathrm{P}_{\mathrm{PT}}$ were highly correlated (fig. $2 \mathrm{~b}$ ). We also observed that as $\%$ area stenosis increased, $\Delta \mathrm{P}_{\mathrm{MR}}$ also increased (fig. 2c).

\section{Conclusions}

Preliminary results suggest that quantification of $\Delta \mathrm{P}_{\mathrm{MR}}$ in a small caliber is feasible. Further technical improvements in higher in-plane and through-plane spatial resolutions and reduction of noise need to be employed, which could potentially help improve the accuracy of the $\Delta \mathrm{P}_{\mathrm{MR}}$ calculations. 
a.

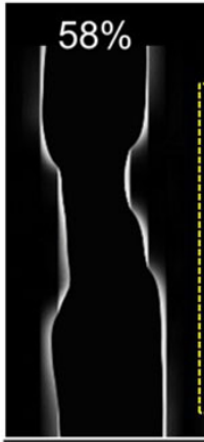

Phantom

(\% area

stenosis)

$58 \%$

c.

$80 \%$

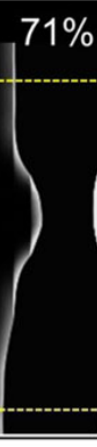

Peak Velocity

(ICC)

0.999

0.891

0.883

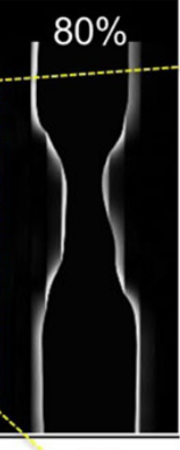

$\Delta \mathrm{P}_{\mathrm{MR}}$

(iCC)

0.950

0.935

0.7673 b. Phase contrast cross-sections $\left(V_{z}\right): 71 \%$ area stenosis

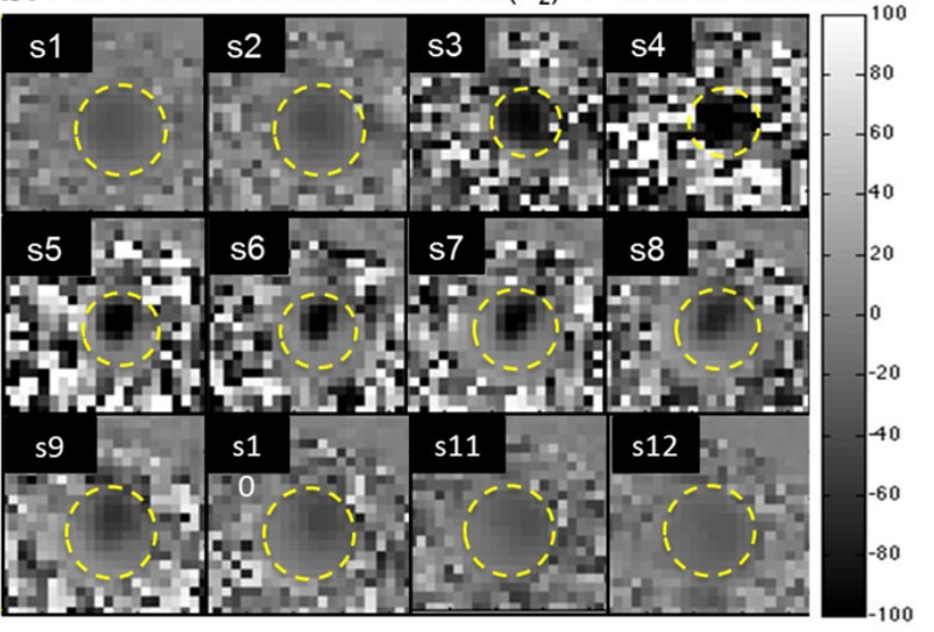

Figure 1 a Stenotic phantom model examples (\% area stenosis at the maximum narrowing). b 2D PC-MRI images in the through-plane direction (velocity maps, $\mathrm{cm} / \mathrm{s}$ ) for $71 \%$ area stenosis phantom model. c Intra-class correlation coefficients (ICC) of the peak velocities and $\Delta \mathrm{P}_{\mathrm{MR}} \mathrm{S}$ for the example phantom models.

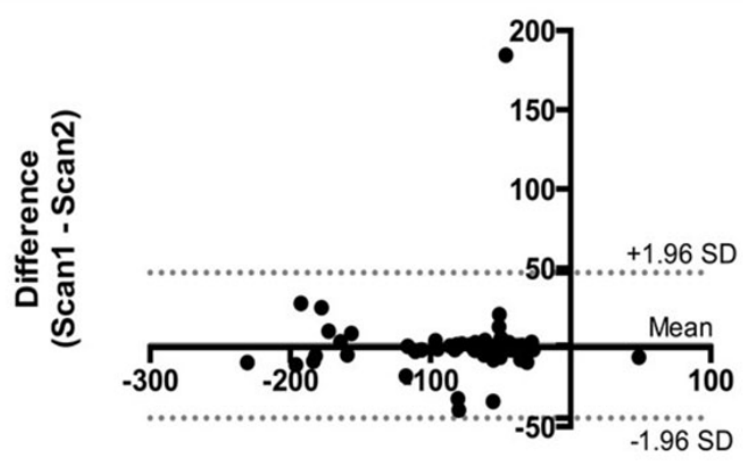

Peak Velocity $(\mathrm{cm} / \mathrm{s})$

a. (Scan1 + Scan2 / 2)

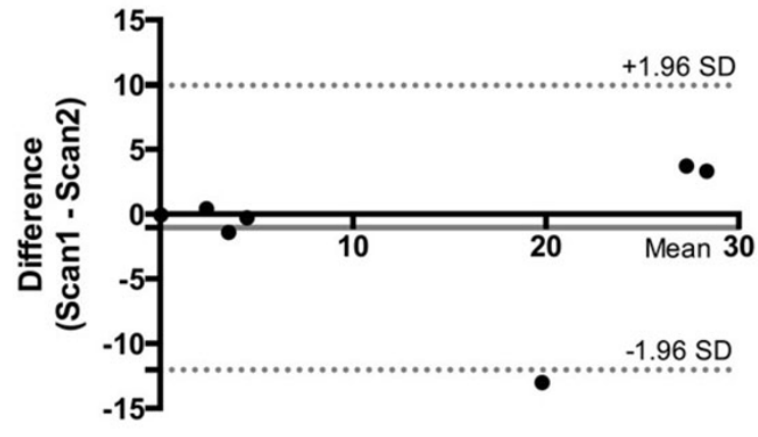

Pressure Difference via NS-calculation $(\mathrm{mmHg})$ (Scan1 + Scan2 / 2)

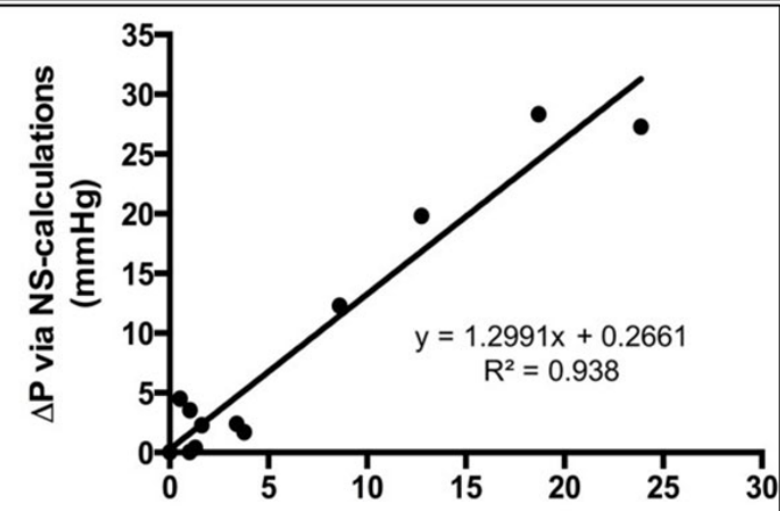

b.
$\Delta \mathrm{P}$ via Pressure-transducer $(\mathrm{mmHg})$

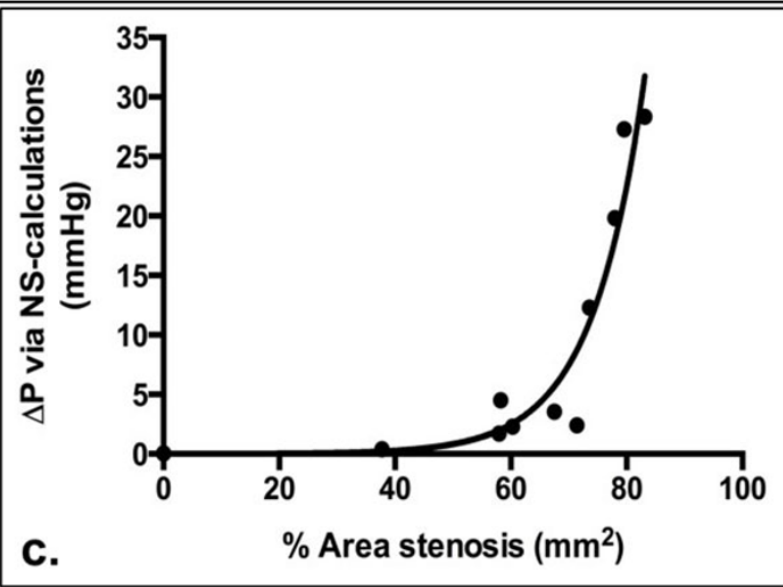

Figure 2 a Bland-Altman plots of peak velocities at all cross-sectional slice from repeat PC-MRI scans and the derived $\triangle \mathrm{P}$ of the phantom models. Mean (bias) and $95 \%$ confidence internal limits are displayed. b Pressure measurement comparison between $\triangle \mathrm{P}$ calculated via NS-equations and $\Delta P$ measured using pressure transducer. Excellent correlation $\left(R^{2}=0.938\right)$ was observed between the two techniques. c $\%$ area stenosis versus $\Delta \mathrm{P}_{\mathrm{MR}}$ measurement. An exponential increase in $\Delta \mathrm{P}_{\mathrm{MR}}$ was observed as $\%$ area stenosis increases. 


\section{Authors' details}

${ }^{1}$ Cedars Sinai Medical Center, Los Angeles, CA, USA. ${ }^{2}$ Bioengineering, University of California, Los Angeles, Los Angeles, CA, USA. ${ }^{3}$ Severance Cardiovascular Hospital, Yonsei College of Medicine, Seoul, Korea (the Republic of). ${ }^{4}$ MR R\&D, Siemens Healthcare, Los Angeles, CA, USA.

${ }^{5}$ Radiology, Research Institute of Radiological Science, Yonsei University College of Medicine, Seoul, Korea (the Republic of).

Published: 27 January 2016

\section{References}

1. Pijls, et al: NEJM 1996.

2. Bock, et al: MRM 2011.

3. Lum, et al: RY 2007.

4. Bley, et al: RY 2011.

5. Deng, et al: ISMRM 2014.

6. Yang, et al: MRM 1996.

doi:10.1186/1532-429X-18-S1-W15

Cite this article as: Deng et al:: Pressure gradient measurement using phase contrast (PC)-MRI in stenotic phantom models: Towards noninvasive quantification of fractional flow reserve in the coronary arteries. Journal of Cardiovascular Magnetic Resonance 2016 18(Suppl 1): W15.

\section{Submit your next manuscript to BioMed Central} and take full advantage of:

- Convenient online submission

- Thorough peer review

- No space constraints or color figure charges

- Immediate publication on acceptance

- Inclusion in PubMed, CAS, Scopus and Google Scholar

- Research which is freely available for redistribution

Submit your manuscript at www.biomedcentral.com/submit 